\title{
Efeitos do HIIT no perfil metabólico de idosos com Diabetes Mellitus tipo 2
}

\author{
Effects of HIIT on the metabolic profile of elderly people with Type 2 Diabetes Mellitus \\ Efectos del HIIT en el perfil metabólico de personas mayores con Diabetes Mellitus tipo 2
}

Recebido: 04/02/2021 | Revisado: 13/02/2021 | Aceito: 13/02/2021 | Publicado: 21/02/2021

\author{
Elton Carlos Lima dos Santos \\ ORCID: https://orcid.org/0000-0002-1463-5651 \\ Centro Universitário AGES, Brasil \\ E-mail: eltonsantos.edf@gmail.com \\ Davi Soares Santos Ribeiro \\ ORCID: https://orcid.org/0000-0001-9816-2566 \\ Centro Universitário AGES, Brasil \\ E-mail: profdavi@live.com
}

\begin{abstract}
Resumo
Programas de exercício físico propiciam na atenuação dos efeitos deletérios do envelhecimento, tal como os processos relacionados a resistência à insulina e diabetes mellitus tipo 2 nos idosos. Partindo desse pressuposto, o presente trabalho tem o objetivo de identificar os efeitos do programa HIIT no perfil metabólico de idosos com diabetes tipo 2. $\mathrm{O}$ estudo trata-se de uma pesquisa bibliográfica com abordagem qualitativa. Foi observado que o HIIT atua diretamente no processo de lipotoxicidade, reduzindo níveis de ácidos graxos livres circulantes, bem como, na redução da expressão de adipocinas pró inflamatórias. Conclui-se que o HIIT é uma valiosa ferramenta para o controle/atenuação da resistência à insulina em idosos diabéticos tipo 2.
\end{abstract}

Palavras-chave: Diabetes; Envelhecimento; Treinamento intervalado de alta intensidade.

\begin{abstract}
Physical exercise programs help to mitigate the harmful effects of aging, such as processes related to insulin resistance and type 2 diabetes mellitus in the elderly. Based on this assumption, the present study aims to identify the effects of the HIIT program on the metabolic profile of elderly people with type 2 diabetes. The study is a bibliographic research with a qualitative approach. It was observed that HIIT acts directly in the lipotoxicity process, reducing levels of free circulating fatty acids, as well as, in reducing the expression of pro-inflammatory adipokines. It is concluded that HIIT is a valuable tool for the control / attenuation of insulin resistance in elderly type 2 diabetics.
\end{abstract}

Keywords: Diabetes; Aging; High-intensity interval training.

\begin{abstract}
Resumen
Los programas de ejercicio físico ayudan a mitigar los efectos nocivos del envejecimiento, como los procesos relacionados con la resistencia a la insulina y la diabetes mellitus tipo 2 en los ancianos. Partiendo de este supuesto, el presente estudio tiene como objetivo identificar los efectos del programa HIIT sobre el perfil metabólico de las personas mayores con diabetes tipo 2. El estudio es una investigación bibliográfica con enfoque cualitativo. Se observó que el HIIT actúa directamente en el proceso de lipotoxicidad, reduciendo los niveles de ácidos grasos libres circulantes, así como en la reducción de la expresión de adipocinas proinflamatorias. Se concluye que el HIIT es una herramienta valiosa para el control / atenuación de la resistencia a la insulina en los diabéticos de tipo 2 de edad avanzada.
\end{abstract}

Palabras clave: Diabetes; Envejecimiento; Entrenamiento de intervalos de alta intensidad.

\section{Introdução}

O envelhecimento populacional é uma realidade a ser refletida pelos profissionais de saúde. Segundo as Nações Unidas, a população em meados de 2015 atingiu o patamar de 7,3 bilhões de pessoas, onde cerca de 12\% que corresponde a aproximadamente 901 milhões de pessoas possuíam idade igual ou superior a 60 anos (UN, 2015). Os dados apresentam que a população mundial só tende a crescer e, possivelmente até o ano de 2030 será atingindo o patamar de 8,5 bilhões de pessoas, sendo que aproximadamente 1,4 bilhões sejam pessoas com idade igual ou superior a 60 anos (UN, 2015).

Deste modo, pode-se inferir que essa revolução demográfica é algo inevitável, cabe aos profissionais de saúde criar estratégias para amenizar os efeitos deletérios desse processo. Segundo Macena, et al. (2018), os processos deletérios do 
envelhecimento favorecem o desenvolvimento de alterações no sistema imunológico, endócrino, neurológico e muscular, e assim, corrobora para o aparecimento de doenças fisiológicas como o diabetes mellitus que é uma das principais causas de internações hospitalares e morte na parcela idosa da população (Roseto, 2019).

$\mathrm{O}$ diabetes mellitus atualmente apresenta crescimento semelhante ao crescimento populacional. Segundo $\mathrm{CHO}$, et al. (2018), estima-se que cerca de $8,8 \%$ da população mundial, equivalente a 424,9 milhões de pessoas, entre 20 e 79 anos viviam com diabetes no ano de 2017, sendo que até 2045, cerca de 628,6 milhões de pessoas irão desenvolver essa doença.

Tendo em vista o grande quantitativo de casos de diabetes mellitus em todo mundo e suas projeções futuras, fica evidente que se trata de um problema grave em âmbito mundial, que chama atenção, principalmente, devido ao crescimento da representatividade dessa patologia na parcela idosa da população. De acordo com Prado, et al. (2016), esta patologia está associada a um maior risco de morte prematura e a outras comorbidades, a priori, as relacionadas com as principais síndromes geriátricas como poli medicação, fragilidade e deficiência cognitiva.

O diabetes mellitus tipo 2 (DM2) é a forma mais comum do diabetes mellitus, porém existem outros tipos, como o diabetes mellitus tipo 1 (DM1) e o diabetes gestacional. Contudo, segundo Colberg, et al. (2016), o diabetes mellitus tipo 2 prevalece em cerca de $90 \%$ a $95 \%$ dos casos registrados e resulta na combinação da incapacidade de a célula muscular responder apropriadamente a insulina, tal como, uma secreção insulínica inadequada.

Segundo a Sociedade Brasileira de Diabetes (2019), a prevalência do diabetes mellitus nos idosos está veemente associada a disfunção da célula beta, com menor produção da insulina e uma maior resistência a mesma, porém, também relacionadas a alterações corporais advindas do envelhecimento. As duas formas mais efetivas para o tratamento do diabetes mellitus são os medicamentos e a atividade física, porém segundo Prado (2016), os idosos conhecem na maioria dos casos apenas o tratamento farmacológico, e assim, não costumam praticar atividades físicas regulares, que de acordo com Colbert, et al. (2016) é essencial para o controle da glicemia em indivíduos com diabetes e pré-diabetes.

No que se refere a prática de atividade física, o método High Interval Intense Training (HIIT) vem se mostrando uma metodologia promissora atenuar os efeitos dessa patologia. De acordo com Tijonna, et al. (2013), o método HIIT consiste na alternância de períodos de alta intensidade com intervalos de recuperação passiva ou ativa em uma intensidade menor, geralmente moderada à baixa. Os protocolos HIIT trazem através de adaptações fisiológicas (cardiovasculares, músculo metabólicas, respiratórias e neurais) e adaptações psicológicas (motivação, percepção de esforço e humor) inúmeros benefícios a saúde (Gibala, 2007).

Nesse contexto, o presente estudo tem como objetivo identificar os efeitos do HIIT no perfil metabólico de idosos com diabetes tipo 2 .

\section{Metodologia}

O estudo trata-se de uma pesquisa bibliográfica com método qualitativo. Segundo Boccato (2006), a pesquisa bibliográfica busca a resolução de um problema através de referenciais teóricos publicados. O método qualitativo, segundo Pereira (2018), é aquele no qual o pesquisador interpreta e opina sobre o fenômeno em estudo.

Para realizar o levantamento bibliográfico foram utilizados livros e artigos científicos encontrados nas bases de dados. Utilizou-se os descritores: Diabetes, Envelhecimento, HIIT e Resistência à insulina. Foram considerados os seguintes parâmetros limitadores da busca inicial: a) artigos publicados entre 1959 e 2020; b) redigidos em língua portuguesa, espanhola ou inglesa; e, c) publicações que tivessem como foco a temática do estudo.

Após levantamento preliminar nas bases de dados escolhidas, os resumos dos estudos selecionados foram revisados de modo a poder refinar a escolha final das publicações que compõem o corpus deste estudo. 


\section{Resultados e Discussão}

\subsection{HIIT e suas características}

A primeira publicação que descrevia o treinamento intervalado em um jornal científico foi realizada em 1959 por Reindell e Roskamm. Em 1960, um grupo de pesquisa de Irma e Astrand publicaram estudos relacionados ao treinamento intervalado visando entender as respostas agudas de lactato, consumo de oxigênio e frequência cardíaca. Essas primeiras publicações remetiam sobre o treinamento intervalado voltado apenas para melhoria do desempenho (Reindell e Roskamm, 1959; Astrand, et al., 1960).

Com o passar dos tempos o foco das publicações foram ganhando novas vertentes e vem sendo publicados trabalhos voltados para atividades recreativas e jogos de equipe. Cabendo ressaltar a grande importância que vem se dando a esse tipo de treinamento, principalmente devido suas formas de aplicação não apenas para pessoas saudáveis, mas para pessoas com algum tipo de patologia em programas de reabilitação (Helgerud, et al., 2007; Tschakert \& Hoffman, 2013; Gibala, 2018).

Existem inúmeras definições sobre treinamento intervalado de alta intensidade. Para Gibala (2018), o HIIT é comumente definido como episódios relativamente intensos de exercícios que estimulam $>80 \%$ da frequência cardíaca máxima, sendo intercalado por períodos de recuperação através de exercícios de menor intensidade ou descanso. Do mesmo modo, para Tijonna, et al. (2013), o método HIIT não é novo e consiste na alternância de períodos de alta intensidade com intervalos de recuperação ativa ou passiva em uma intensidade geralmente moderada-baixa em cicloergômetros ou tapetes rolantes.

Outrossim, mesmo com essas evidências que demonstram a aplicabilidade e pesquisas sobre o HIIT a mais de 50 anos, ainda existem interpretações equivocadas acerca dessa metodologia. Segundo Del Vecchio (2014), dentre os principais equívocos sobre essa metodologia a principal é sobre sua origem, pois muitos acreditam erroneamente que se trata de uma metodologia contemporânea ou até mesmo que é tema de estudos somente atuais.

Independente do conceito ou origem da metodologia, é um consenso que o treinamento intervalado de alta intensidade traz através de adaptações fisiológicas (cardiovasculares, músculo metabólicas, respiratórias e neurais) e adaptações psicológicas (motivação, percepção de esforço e humor) inúmeros benefícios a saúde, devendo-se ressaltar dentre essas adaptações a melhora do perfil metabólico dos indivíduos (Gibala, 2018).

Para se atingir essas benesses faz-se necessário entender que diferentemente do exercício contínuo que compreende somente a intensidade da carga de trabalho e a duração total, o HIIT possui cinco componentes primordiais, sendo eles o pico da carga de trabalho (pico $\mathrm{P}$ ), a duração do pico da carga de trabalho (pico $\mathrm{T}$ ), carga da recuperação ( $\mathrm{P}$ rec.), duração da recuperação ( $\mathrm{T}_{\text {rec. }}$ ) e a carga média ( $\mathrm{P}_{\text {média }}$ (Tschakert \& Hoffman, 2013).

Na Imagem 1, Buchheit e Laursen (2013) descomplicam as variáveis para a elaboração do programa HIIT trazendo conceitos como a intensidade que é a quantidade de trabalho executado, a modalidade a ser executada, o tipo de HIIT escolhido para a sessão e o número de séries, bem como, o tempo de intervalo entre as séries, que juntos influenciam na intensidade média da sessão. 
Imagem 1: Variáveis para a elaboração de uma sessão HIIT.

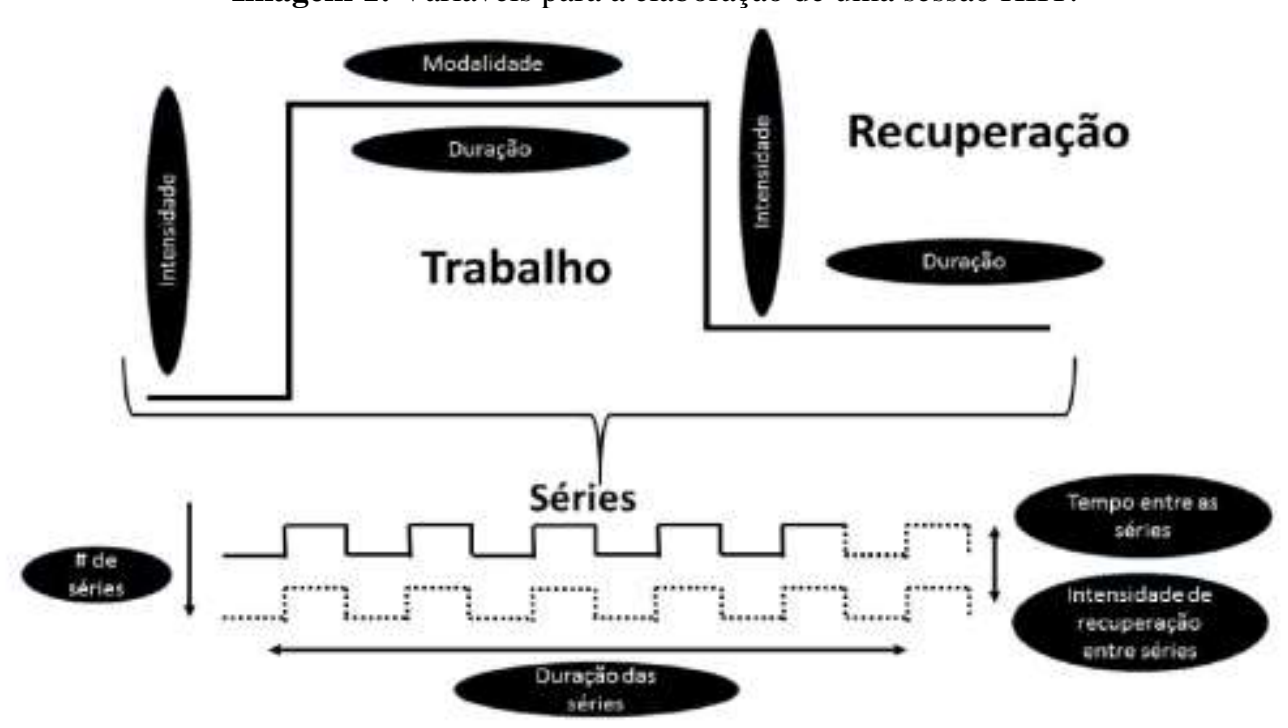

Fonte: Buchheit e Laursen (2013).

Dessa forma, a partir da manipulação desses componentes, pode-se prescrever diferentes tipos de HIIT, que são separados em quatro grandes grupos, sendo o primeiro o HIIT-curto, que é composto por treinos nos quais os esforços duram menos que 1 minuto e a relação esforço-pausa apresentam valores próximos. O segundo é o HIIT-longo, que os esforços duram acima de 1 minuto e a relação esforço-pausa apresentam valores próximos, o terceiro é o SIT, que se trata de um treinamento intervalado de alta intensidade baseado em sprints e geralmente o período de esforço é curto em intensidade All-Out e o descanso é longo. Por fim, o RST que compreende um trabalho de sprints repetitivos de curta duração com pausas também curtas (Del Vecchio, 2014).

Os períodos de pausa dessa forma de treinamento podem ser realizados de forma passiva, onde o indivíduo recuperase esperando parado até o próximo esforço, ativa livre, onde o indivíduo descansa se movimentando de forma auto selecionada, e ativa controlada que concerne em uma recuperação a uma intensidade determinada (Tschakert \& Hoffman, 2013; Del Vecchio, 2014).

O HIIT deve abranger alguns desses parâmetros: está acima de 15 na escala de percepção do esforço, acima de $85 \%$ da frequência cardíaca máxima medida ou predita, acima de $85 \%$ da intensidade associada ao consumo máximo de oxigênio ou deve estar acima do anaeróbico medido por teste de esforço (Del Vecchio, 2014). Assim, pode-se controlar o período de esforço do HIIT através de parâmetros fisiológicos, como a frequência cardíaca máxima e/ou de reserva, concentração sanguínea de lactato, percepção subjetiva de esforço ou consumo máximo de oxigênio, entre outros.

\subsection{Efeitos Fisiológicos do HIIT no Controle Glicêmico}

O HIIT vem sendo alvo de pesquisas e atualmente graças as suas adaptações agudas e crônicas é uma ferramenta de grande valia para o Profissional de Educação Física (Colberg, et al., 2016). As respostas agudas após a sessão do treinamento HIIT permeiam interações biomoleculares a níveis da AMPK, GLUT-4 e captação de glicose. As respostas moleculares agudas após uma sessão HIIT está intimamente ligada a fosforilação da cinase dependente de AMP (AMPK), qual é dividida em 3 subunidades, sendo elas ALFA, BETA e GAMA. Cada uma pode ser encontrada em maior ou menor quantidade a depender do tecido, e podem regular a ativação da AMPK de formas diferentes (Gomes, 2017).

A ativação de AMPK segundo Zhang e Zhou (2009) exerce um grande impacto no metabolismo celular, sendo essencial para a homeostase da glicose e, portanto, é um alvo farmacológico para reverter as anormalidades metabólicas como 
a resistência à insulina. A ativação da AMPK tem efeitos em uma gama de tecidos, cabendo ressaltar seus efeitos no músculo esquelético, onde estimula a captação da glicose, oxidação de ácidos graxos, translocação de GLUT-4 e biogênese mitocondrial, enquanto inibe a síntese de proteína e glicogênio e no fígado que estimula a captação de glicose e oxidação dos ácidos graxos, enquanto inibe a gliconeogênese hepática (Colghlan, 2014; Gomes, 2017).

Colghlan (2014) aponta que quase todos os efeitos da ativação da AMPK seriam benéficos para pacientes com diabetes tipo 2, pois, pode ser observado em animais e humanos que apresentam síndrome metabólica e/ou diabetes tipo 2 que a AMPK se apresenta desregulada. Dessa forma, corroborando com Zhang e Zhou (2009), numerosos agentes farmacológicos buscam induzir a ativação da AMPK, cabendo ressaltar dentre eles a metformina, já citada anteriormente neste trabalho.

Contudo, foi verificado que as isoformas da subunidade GAMA podem ser encontradas em maior quantidade nas fibras musculares tipo 2 e são extremamente sensível a razão AMP/ATP, permitindo assim, destacar que essa subunidade é altamente responsiva a hidrólise de ATP proveniente ao exercício físico de alta intensidade e curta duração como o HIIT. Assim, pode-se inferir que o HIIT é uma alternativa não medicamentosa para o controle da homeostase glicêmica em diabéticos tipo 2 (Carling, 2004).

Segundo Gomes (2017), a cascata de fosforilação da AMPK em resposta ao treinamento HIIT parece ter maior influência na fosforilação da acetil-Coa Carboxilase (ACC), da TBC1D1, TBC1D4 e da proteína quinase ativada por mitógeno p38 (p38 MAPK), o que potencializa o transporte de ácidos graxos para mitocôndria pela carnitina palmitoil transferase 1 (CPT-1), bem como a translocação de GLUT-4 para membrana da célula, e assim, afeta positivamente a captação da glicose independente de insulina.

A ativação de AMPK modula positivamente o Coativador - 1 alfa do receptor ativado por proliferador de peroxissoma (PGC-1 alfa), que induz a expressão de genes como o Piruvato desidrogenase quinase (Pdk4) e Carnitina Palmitoil transferase 1b (CPT-1b) e assim, contribui positivamente para a biogênese mitocondrial, tal como, a expressão do gene associado ao GLUT-4.

Corroborando com esse fato, estudo de Za'don, et al. (2019) identificou em um grupo com 25 indivíduos submetidos a 12 semanas de HIIT (três vezes por semana), a multiplicação de aproximadamente três vezes na expressão de PGC1-alfa, bem como, uma melhora significativa na resistência à insulina desse grupo, e assim, demonstrando um efeito crônico do HIIT (Za'don, et al., 2019).

Como citados anteriormente, o HIIT também proporciona adaptações crônicas que concernem em apropriação fisiológica das adaptações agudas do treino, e assim, são efeitos permanentes ao indivíduo. Scribbans, et al. (2014), ao comparar um protocolo aeróbico contínuo versus o protocolo HIIT, ambos em bicicleta, identificou que ambos os protocolos aumentavam significativamente a atividade da enzima glicerolfosfato desidrogenase, que converte diidroxiacetona-fosfato e gliceraldeído-3-fosfato em fibra tipo 1, porém apenas o protocolo HIIT aumenta essa expressão nas fibras tipo 2, o que sugere que o HIIT pode influenciar positivamente a longo prazo na capacidade glicolítica.

Do mesmo modo, Terada et al. (2001), em protocolos distintos (HIIT versus contínuo) de 8 semanas com ratos Sprague Dawley, constatou que no músculo epitroclear dos animais de ambos os protocolos houve um aumento de GLUT-4 e maior transporte de glicose mediado pela insulina, e assim, sugerindo que ambos protocolos são capazes de gerar adaptações crônicas que possibilitam melhorar o transporte de glicose.

Umas das principais adaptações promovida pelo HIIT é o aumento do da expressão de PGC-1 alfa no decorrer das sessões que acarreta um aumento crônico dessa proteína, e assim, influência de forma positiva e direta a capacidade oxidativa. Essa proteína sofre uma down-regulation em indivíduos resistentes a insulina, e assim, pode-se inferir que o aumento da expressão gênica dessa proteína é uma chave para a melhora da sensibilidade a insulina no músculo esquelético via aumento da densidade mitocondrial e expressão de GLUT-4 (Santos, et al., 2014). 
Em estudo experimental de Maleki, et al. (2018), com 24 ratos wistar machos divididos aleatoriamente em 4 grupos (saudável, saudável controle, obeso e obeso controle) e submetidos a natação HIIT por seis semanas, foi constatado a diminuição do peso corporal dos ratos obesos treinados e menor metilação de PGC-1 alfa dos treinados em comparação ao controle, o que sugere a ocorrência de uma maior expressão de PGC-1 alfa induzido pelo treinamento HIIT (Maleki, 2018).

O estudo de Chavanelle, et al. (2017), buscou comparar os efeitos do treinamento contínuo versus o HIIT sobre o metabolismo da glicose e função mitocondrial em camundongos diabéticos. Como resultado o trabalho revelou um aumento de cerca de 2 vezes no conteúdo de GLUT-4 muscular e maiores razões de fosforilação de Akt estimulada pela insulina no grupo HIIT. Portanto, tendo em vista que tanto a AMPK quanto o PGC1-alfa são precursores da maior expressão de GLUT-4. Bem como, maior biogênese mitocondrial, levando em conta que as defasagens dessas vias são associadas a resistência à insulina, pode-se deduzir que a expressão dessas proteínas via exercício é de extrema valia para a atenuação da diabetes tipo 2 (Chavanelle, et al., 2017).

Em relação a remodelamentos das fibras musculares, Gomes (2017) constatou a capacidade do HIIT em promover remodelamento das fibras musculares no sentido IIB > IIA > IA, que vai de encontro as adaptações biomoleculares desse treinamento, mas que, por outro lado resulta em maior quantidade oxidativa, que é benéfico para pessoas com diabetes tipo 2 .

Por fim, a partir dos pressupostos acerca das alterações biomoleculares induzidas pelo HIIT de forma aguda e crônica é plausível a ideia de que o HIIT pode influenciar positivamente na questão da resistência à insulina, uma vez que a dificuldade na captação da glicose é o principal problema desse quadro.

\subsection{Efeitos do HIIT na Expressão das Adipocinas}

O tecido adiposo, principalmente o visceral, é um tecido ativo que libera substâncias denominadas adipocinas, que corroboram para a instalação do quadro de resistência à insulina e, por conseguinte, o diabetes mellitus tipo 2. A partir disso, esse subtópico busca analisar a influência do HIIT na supressão dessas adipocinas. Pois, a supressão da inflamação parece ser uma boa abordagem terapêutica para diminuir o estado inflamatório induzido pela obesidade e distúrbios imunometabólicos (Soltani, et al., 2020).

Partindo desse pressuposto, Banitalebi, et al. (2018) em seu estudo analisou a influência de 10 semanas de treinamento combinado (resistido + contínuo) versus o HIIT na expressão das adipocinas inflamatórias em um grupo de mulheres com diabetes tipo 2. Como resultado, foi constatado que as concentrações de TNF-alfa reduziram significativamente no grupo HIIT e no treinamento combinado, contudo foram encontradas diferenças significativas em relação a IL-6 apenas no grupo HIIT. O que sugere que ambos os protocolos são benéficos para essa atenuação, porém mostrando-se mais promissor o HIIT.

Em estudo que buscou analisar um grupo de 28 mulheres com sobrepeso divididas aleatoriamente em um subgrupo controle e um subgrupo HIIT durante 8 semanas (três sessões por semana) foi identificado uma redução significativa no grupo treinado em comparação ao grupo controle na expressão de TNF-alfa, bem como, melhora no perfil lipídico, IMC e percentual de gordura total. Sugere-se que o HIIT é uma importante ferramenta para o controle do peso corporal e atenuação da inflamação crônica advinda do sobrepeso (Taherichadorneshin, et al., 2019).

Do mesmo modo, Gerosa-Neto, et al. (2016), buscando avaliar o efeito três protocolos de treinamento (contínuo longo versus contínuo curto $1 \times 4$ minutos versus HIIT $4 \times 4$ minutos) em 35 indivíduos durante 16 semanas, sobre o perfil inflamatório, resistência à insulina e sobrepeso. Obteve como resultado uma diminuição nas concentrações de IL-6 no grupo HIIT e uma diminuição do TNF-alfa no grupo contínuo, contudo foi observado um leve aumento de TNF-alfa no grupo HIIT. Em relação a adiponectina houve uma diminuição nos três modelos de treinamento. Apesar do aumento de TNF-alfa e a 
diminuição da adiponectina, houve tendência a diminuição do IMC e melhora da sensibilidade a insulina no grupo HIIT, o que foi de encontro aos achados na pesquisa (Gerosa-Neto, et al., 2016).

Outro estudo que cabe ressaltar neste trabalho, é o de Soltani, et al. (2020), que buscou avaliar a influência do HIIT no estado inflamatório em mulheres sedentárias e obesas durante 10 semanas. Como resultado, foi identificado que os níveis de TNF-alfa foram reduzidos significativamente, além de ser observado uma correlação negativa entre a adiponectina e os valores da insulina. A partir disso, o estudo sugere que o aumento da sensibilidade da insulina advindo do HIIT nesses quesitos avaliados concerne em uma diminuição do estado inflamatório advindo pela diminuição de TNF-alfa, tal como, o aumento da adiponectina que é conhecido por ser um sensibilizador da insulina.

Por fim, com base nas alterações analisadas percebe-se que o HIIT é uma ferramenta de grande valia para o profissional de Educação Física atenuar a expressão desses fatores inflamatórios. Pois, a IL-6 prejudica a fosforilação dos receptores de insulina através de diversos caminhos, sendo um deles através da indução da expressão do supressor de sinalização de citocina 3 (SOCS-3), do mesmo modo, o TNF-alfa quando possui expressão exacerbada diminui a fosforilação dos substratos do receptor da insulina (IRS-1 e IRS-2) via c-jun-terminal kinase (JNK), e assim, a partir da atenuação desses dois fatores, pode-se inferir que o HIIT é eficiente para atenuar o processo de inflamação crônica e, por conseguinte, a resistência à insulina.

Contudo, foi demonstrado no estudo de Gerosa-Neto, et al. (2016), que o treinamento HIIT pode induzir a uma supressão da adiponectina, que exerce papel antagônico as adipocinas inflamatórias como TNF-alfa, porém, essa supressão parece não ter sido suficientemente prejudicial a ponto de pôr em dúvida as benesses nas questões inflamatórias propiciadas pelo treinamento HIIT.

\subsection{HIIT e Idosos}

Existem riscos associados a prática do exercício físico, a priori, quando este é realizado com idosos que normalmente possuem mais de uma patologia. Contudo, as benesses advindas do exercício se mostram mais que suficientes para a que sua prescrição seja recomendada para essa população.

Em um estudo de Hwang, et al. (2019), com 58 indivíduos diabéticos e sedentários entre 46 a 78 anos, foi observado que tanto o HIIT quanto o exercício de moderada intensidade são mais seguros e eficazes em um ergômetro de suporte para o corpo inteiro, quando comparados a mesma prática somente na esteira para idosos previamente sedentários e com diabetes tipo 2, a priori, quando estes têm dificuldades para participar de exercícios com levantamento de pesos.

Dentre as benesses atualmente mais estudadas propiciadas pelo HIIT para essa população, os aspectos relacionados com a resistência à insulina e o diabetes tipo 2 estão no topo. No Quadro 1 é possível acompanhar uma análise dos efeitos do HIIT nos idosos: 
Quadro 1: Análise dos efeitos do HIIT no perfil metabólico de idosos.

\begin{tabular}{|c|c|c|c|c|}
\hline Autor & População & Idade & Descrição & Resultado \\
\hline Lithgow e Leggate, 2018 & Idosos não diabéticos & $64 \pm 2$ & $\begin{array}{l}\text { Sessão única } \\
\text { HIIT: aquecimento } 5 \text { min. A } 50 \mathrm{~W} \text {; work } \\
10 \text { intervalos de } 1 \mathrm{~min} \text { a } 100 \% \text { do vo } 2 \\
\text { pico, intercalada a } 1 \mathrm{~min} \text { a } 50 \mathrm{~W} \text {. }\end{array}$ & $\begin{array}{l}\text { Glicose jejum } \downarrow \\
\text { Insulina jejum } \downarrow \\
\text { HOMAR-IR } \uparrow \\
\text { IL-6 } \uparrow \\
\text { TNF-alfa } \downarrow \\
\text { Colesterol } \downarrow \\
\text { Triglicerídeos } \downarrow\end{array}$ \\
\hline Hayes, et al., 2020 & 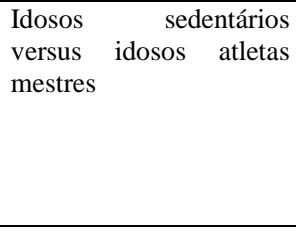 & $60 \pm 5$ & $\begin{array}{l}6 \text { semanas de treinamento intervalado } \\
\text { de alta intensidade }(6 \times 30 \text { s sprint a } 40 \% \\
\text { da potência de pico, uma vez a cada } 5 \\
\text { dias }\end{array}$ & $\begin{array}{l}\text { Sedentários: Insulina } \downarrow \\
\text { Glicose jejum } \downarrow \\
\text { HOMAR-IR } \downarrow \\
\text { Mestres: } \\
\text { Insulina } \uparrow \\
\text { Glicose } \uparrow \\
\text { HOMA-IR } \downarrow \\
\end{array}$ \\
\hline Mitranun, et al., 2013 & Idosos diabéticos tipo 2 & $50-70$ & $\begin{array}{l}\text { Total de } 12 \text { semanas envolvendo } \\
\text { exercícios de esteira } 3 \times / \text { semana. } \\
\text { O programa era } \\
\text { dividido em } 3 \text { fases. } 1^{\text {a }} \text { fase, } 50 \% \text { da } \mathrm{HR} \\
\text { a } 30^{\prime} .2^{\circ} \text { estágio, aquecimento } 5^{\prime} \text { a } 50 \% \\
\text { do FC após } 4^{\prime} \text { para } 80 \% \text { FC com } \\
\text { repouso de } 4^{\prime} \text { a } 50 \% \text { FC para } 20^{\prime} \text { e }+5^{\prime} \\
\text { de volta para calma. } 3^{\circ} \text { estágio, } 60 \% \mathrm{FC} \\
\text { por 5', após } 1^{\prime} \text { para } 85 \% \text { FC por } 6 \\
\text { intervalos com } 4^{\prime} \text { a } 60 \% \text { FC resto. } \\
\text { Retorna para acalmar } 5^{\prime} \text { a sessão durou } \\
40^{\prime} \text {. }\end{array}$ & $\begin{array}{l}\text { Glicose jejum } \downarrow \\
\text { Resist. insulina } \downarrow \\
\text { HbA1c } \downarrow\end{array}$ \\
\hline Mailarrd, et al., 2016 & $\begin{array}{l}\text { Melhores pós } \\
\text { menopausa com diabetes } \\
\text { tipo } 2\end{array}$ & $61-80$ & $\begin{array}{l}\text { Total de } 16 \text { semanas ( } 2 \text { x semana) em } \\
\text { bicicletas, aquecimento 5', seguido por } \\
\text { seguido por } 12 \text { " de intervalos de } \\
\text { recuperação pedalando de } 20 \text { a } 30 \text { rpm. } \\
\text { Máximo de } 60 \text { ciclos de } 20 \text { ' sessão, } \\
\text { retido a calma 5'. }\end{array}$ & $\begin{array}{l}\text { Controle glicêmico } \uparrow \\
\text { Resistência Insulina } \downarrow \\
\text { Pressão sanguínea } \downarrow \\
\text { Capacidade aeróbica } \uparrow\end{array}$ \\
\hline Hollekin, et al., 2014 & $\begin{array}{l}\text { Idosos com diabetes tipo } \\
2\end{array}$ & $58,6 \pm 5$ & $\begin{array}{l}\text { Total de } 12 \text { semanas }(3 \text { vezes por } \\
\text { semana). Em esteira com inclinação } \\
\text { realizou-se } 4 \text { x } 4 \text { min a 90 95\% da FC } \\
\text { max }\end{array}$ & $\begin{array}{l}\text { HOMA-IR } \uparrow \\
\text { HbAlc } \downarrow \\
\% \text { gordura } \downarrow \\
\text { IMC } \downarrow\end{array}$ \\
\hline
\end{tabular}

Fonte: Autores (2021)

Lithgow e Leggate (2018) com o intuito de investigar se uma única sessão de HIIT seria capaz de promover alterações na tolerância a glicose e outros marcadores fisiológicos e metabólicos em idosos não diabéticos (64 \pm 2 ) submeteu um grupo de idosos a uma sessão de exercício contínuo moderado e outro grupo a uma sessão de HIIT. Como resultado principal foi observado que a concentração de insulina após o OTG foi aumentada em 60 minutos em comparação com o controle, porém não houve mudanças significativas nos níveis de glicose. Outrossim, foi observado um potencial dessa metodologia em reduzir a inflamação crônica advinda das adipocinas inflamatórias

Do mesmo modo, em um estudo de caráter mais crônico, Hayes (2020) investigou se 6 semanas de treinamento intervalado de alta intensidade ( 6 x 30s sprint a $40 \%$ da potência de pico, uma vez a cada 5 dias) após 6 semanas de pré condicionamento aeróbico, seria capaz de promover melhora na insulina em jejum, glicose e homeostatic model assessment (HOMAR-IR), que calcula a relação da quantidade de glicose e insulina no sangue, em indivíduos sedentários mais velhos (62 \pm 2 ) comparado com atletas mestres $(60 \pm 5)$. Como resultado, foram observadas evidências preliminares que sugerem que o HIIT precedido de pré condicionamento promove pequenas melhorias na insulina em jejum, glicose e HOMAR-IR em idosos sedentários, quando comparados a atletas mestres. Sugerindo, portanto, que as alterações dos níveis glicêmicos nos idosos estão associadas aos benefícios crônicos dessa metodologia.

Corroborando com esse achado, Mitranun, et al. (2013) em um estudo com idosos diabéticos (50-70), observou que 12 semanas de um protocolo de HIIT foi capaz de induzir melhorias significativas na glicose em jejum, resistência à insulina $\mathrm{e}$ HbA1c1.O resultado advindo de forma crônica mediado pelo HIIT, também foi evidenciado no estudo de Mailarrd, et al. (2016), que semelhante aos dois estudos anteriores, identificou que um programa longo de HIIT (16 semanas) é capaz de 
promover melhorias significativas no controle glicêmico e resistência à insulina em mulheres idosas pós menopausa (61-80). Outrossim, também foi evidenciado resultados semelhantes no estudo de Hollekin, et al. (2014), que ao submeter idosos com diabetes tipo 2 a um programa de 12 semanas com um total de 36 sessões de HIIT, apresentou resultados que demonstravam a eficiência do HIIT em reduzir o índice HOMA-IR, a HbA1c, o \% gordura e o IMC dessa população.

É evidente que o HIIT é seguro e efetivo para o controle glicêmico de idosos. Contudo, como já foi discutido nesse capítulo, as variáveis do HIIT podem alterar o tipo de treinamento, mas comumente, dividido em curto e longo. A partir disso, Golshan, et al. (2019), em seu estudo buscou analisar o baixo volume versus o alto volume de HIIT em diabéticos tipo 2 . Onde, o HIIT curto teve como intensidade máxima até $110 \%$ da FC e o HIIT longo teve como intensidade máxima até $80 \%$ da FC, com práticas de 3 dias por semana durante 8 semanas.

Como resultado, foram observados que ambos os grupos tiveram diferença significativa em comparação ao grupo controle. Contudo, o grupo do baixo volume obteve destaque em diminuir significativamente os níveis de glicose, HbA1c, insulina, resistência à insulina e triglicerídeos, apontando que essa forma de HIIT se mostra mais promissora como alternativa não farmacológica de controle glicêmico.

\section{Conclusão}

Ao analisar os efeitos HIIT no perfil inflamatório de idosos com Diabetes mellitus tipo 2, observaram-se reduções significativas no estado inflamatório induzido pelo acúmulo excessivo de tecido adiposo nos indivíduos submetidos a protocolos de HIIT, a priori, na expressão do TNF-alfa e IL-6. Entretanto, foram observados em alguns estudos que o HIIT corrobora para a redução do nível de adiponectina, porém sem interferência significativa na redução do processo inflamatórias proporcionado pelo HIIT.

Ademais, ao analisar a relação do HIIT com a melhora do perfil metabólico dos idosos, foram observadas melhoras significativas em todos os aspectos correlacionado ao controle glicêmico na população idosa, inclusive em índices correlacionados a lipotoxicidade como os triglicerídeos, colesterol e excesso de peso, sendo mais expressivas em programas de HIIT com baixo volume.

Por fim, partindo desses pressupostos, pode-se inferir que o treinamento intervalado de alta intensidade, a priori, em sua forma time-efficient é uma ferramenta de grande valia para o profissional de Educação Física induzir melhoras significativas na qualidade de vida dos idosos diabéticos, desde que, o profissional conheça todos os aspectos inerentes a fisiopatologia dos diabetes e as nuances da prescrição da modalidade. Sugere-se que esse artigo possa contribuir em futuros trabalhos relacionados a metodologia do HIIT aplicada em idosos com diabetes mellitus para a melhora da saúde e qualidade de vida.

\section{Referências}

ÅStrand, I., ÅStrand, P. O., Christensen, E. H., \& Hedman, R. (1960). Intermittent muscular work. Acta Physiologica Scandinavica, $48(3-4), 448-453$.

Banitalebi, E., Faramarzi, M., \& Nasiri, S. (2018). High-Intensity Interval Training Versus Moderate Intensity Combined Training (Resistance and Aerobic) for Improving Insulin-Related Adipokines in Type 2 Diabetic Women. Zahedan Journal of Research in Medical Sciences, 20(10).

Boccato, V. R. C. (2006). Metodologia da pesquisa bibliográfica na área odontológica e o artigo científico como forma de comunicação. Rev. Odontol. Univ. Cidade São Paulo, São Paulo, 18(3), 265-274.

Buchheit, M., \& Laursen, P. B. (2013). High-intensity interval training, solutions to the programming puzzle. Sports medicine, 43(5), 313-338.

Carling, D. (2004). The AMP-activated protein kinase cascade-a unifying system for energy control. Trends in biochemical sciences, $29(1)$, 18-24.

Chavanelle, V., Boisseau, N., Otero, Y. F., Combaret, L., Dardevet, D., Montaurier, C., \& Sirvent, P. (2017). Effects of high-intensity interval training and moderate-intensity continuous training on glycaemic control and skeletal muscle mitochondrial function in db/db mice. Scientific reports, 7(1), 1-10. 
Cho, N., Shaw, J. E., Karuranga, S., Huang, Y., da Rocha Fernandes, J. D., Ohlrogge, A. W., \& Malanda, B. (2018). IDF Diabetes Atlas: Global estimates of diabetes prevalence for 2017 and projections for 2045. Diabetes research and clinical practice, 138, 271-281.

Colberg, S. R., Sigal, R. J., Yardley, J. E., Riddell, M. C., Dunstan, D. W., Dempsey, P. C., ... \& Tate, D. F. (2016). Physical activity/exercise and diabetes: a position statement of the American Diabetes Association. Diabetes care, 39(11), 2065-2079.

Del Vecchio, F. B., Ribeiro, Y. S., Picanço, L. M., \& Galliano, L. M. (2014). Exercício Intermitente: Estado da Arte e Aplicações Práticas: Livro Tabata-HiitTreinamento Intervalado De Alta Intensidade-Treino Aeróbio-Treino Anaeróbio. OMP Editora.

United Nations, Department of Economic and Social Affairs, Population Division (2015). World Population Prospects: The 2015 Revision, Key Findings and Advance Tables. Working Paper No. ESA/P/WP.241.

Gerosa-Neto, J., Antunes, B. M., Campos, E. Z., Rodrigues, J., Ferrari, G. D., Neto, J. C. R., \& Bueno, C. R. (2016). Impact of long-term high-intensity interval and moderate-intensity continuous training on subclinical inflammation in overweight/obese adults. Journal of exercise rehabilitation, $12(6), 575$.

Gibala, M. J. (2007). High-intensity interval training: a time-efficient strategy for health promotion?. Current sports medicine reports, 6(4), $211-213$.

Gibala, M. J. (2018). Interval training for cardiometabolic health: why such a HIIT?. Current sports medicine reports, 17(5), 148-150.

Golshan, H., Toloee, M. E., Abbasi, H., \& Namiranian, N. (2020). Effect of Different HIIT Protocols on the Glycemic Control and Lipids Profile in Men with type 2 diabetes: A Randomize Control Trial. Iranian journal of diabetes and obesity.

Hayes, L. D., Herbert, P., Sculthorpe, N., \& Grace, F. (2020). High intensity interval training (HIIT) produces small improvements in fasting glucose, insulin, and insulin resistance in sedentary older men but not masters athletes. Experimental Gerontology, 140, 111074.

Helgerud, J., Høydal, K., Wang, E., Karlsen, T., Berg, P., Bjerkaas, M., \& Hoff, J. (2007). Aerobic high-intensity intervals improve V• O2max more than moderate training. Medicine \& science in sports \& exercise, 39(4), 665-671.

Hwang, C. L., Lim, J., Yoo, J. K., Kim, H. K., Hwang, M. H., Handberg, E. M., ... \& Christou, D. D. (2019). Effect of all-extremity high-intensity interval training vs. moderate-intensity continuous training on aerobic fitness in middle-aged and older adults with type 2 diabetes: A randomized controlled trial. Experimental gerontology, 116, 46-53.

Lithgow, H. M., \& Leggate, M. (2018). The effect of a single bout of high intensity intermittent exercise on glucose tolerance in non-diabetic older adults. International journal of exercise science, 11(3), 95.

Macena, W. G., Hermano, L. O., \& Costa, T. C. (2018). Alterações fisiológicas decorrentes do envelhecimento. Revista Mosaicum, (27), $223-238$.

Maleki, S., Nazarali, P., Razavi, A., \& Kazemi, F. (2018). Effects of six-week high intensity interval training (HIIT) on PGC $1 \alpha$ methylation in astrocnemius muscle of obese rats. Research in Medicine, 42(4), 196-201.

Mitranun, W., Deerochanawong, C., Tanaka, H., \& Suksom, D. (2014). Continuous vs interval training on glycemic control and macro-and microvascular reactivity in type 2 diabetic patients. Scandinavian journal of medicine \& science in sports, 24(2), e69-e76.

Pereira A. S. et al. (2018). Metodologia da pesquisa científica. UFSM. https://repositorio.ufsm.br/bitstream/handle/1/15824/Lic_Computacao_MetodologiaPesquisa-Cientifica.pdf?sequence=1.

Prado, M. A. M. B. D., Francisco, P. M. S. B., \& Barros, M. B. D. A. (2016). Diabetes em idosos: uso de medicamentos e risco de interação medicamentosa. Ciência \& Saúde Coletiva, 21, 3447-3458.

Reindell, H., \& Roskamm, H. (1959). Ein Beitrag zu den physiologischen Grundlagen des Intervalltrainings unter besonderer Berücksichtigung des Kreislaufes. Editions Médecine et Hygiène.

Santos, J. M., Tewari, S., \& Benite-Ribeiro, S. A. (2014). The effect of exercise on epigenetic modifications of PGC1: The impact on type 2 diabetes. Medical hypotheses, 82(6), 748-753.

SBd, D. (2019). Diretrizes da sociedade brasileira de diabetes 2019-2020.

Soltani, N., Marandi, S. M., Kazemi, M., \& Esmaeil, N. (2020). Meta-inflammatory state and insulin resistance can improve after 10 weeks of combined allextremity high-intensity interval training in sedentary overweight/obese females: a quasi-experimental study. Journal of Diabetes \& Metabolic Disorders, 1 10.

TaheriChadorneshin, H., Cheragh-Birjandi, S., Goodarzy, S., \& Ahmadabadi, F. (2019). The impact of high intensity interval training on serum chemerin, tumor necrosis factor-alpha and insulin resistance in overweight women. Obesity Medicine, 14, 100101

Terada, S., Yokozeki, T., Kawanaka, K., Ogawa, K., Higuchi, M., Ezaki, O., \& Tabata, I. (2001). Effects of high-intensity swimming training on GLUT-4 and glucose transport activity in rat skeletal muscle. Journal of Applied Physiology, 90(6), 2019-2024.

Tjønna, A. E., Leinan, I. M., Bartnes, A. T., Jenssen, B. M., Gibala, M. J., Winett, R. A., \& Wisløff, U. (2013). Low-and high-volume of intensive endurance training significantly improves maximal oxygen uptake after 10-weeks of training in healthy men. PloS one, 8(5), e65382.

Tschakert, G., \& Hofmann, P. (2013). High-intensity intermittent exercise: methodological and physiological aspects. International journal of sports physiology and performance, $8(6), 600-610$.

MK, A. F., Farhanim, I., TO, S. I., Appukutty, M., Salim, N., Farah, N. M. F., \& M1, A. F. (2019). High-intensity interval training induced PGC-1 $\propto$ and AdipoR1 gene expressions and improved insulin sensitivity in obese individuals. The Medical journal of Malaysia, 74(6), 461-467.

Zhang, B. B., Zhou, G., \& Li, C. (2009). AMPK: an emerging drug target for diabetes and the metabolic syndrome. Cell metabolism, 9(5), 407-416. 